\title{
¿Arbitraje Procesal Civil? La necesidad de implantar buenas prácticas en el arbitraje
}

\author{
Fernando Cantuarias Salaverry* \\ Nicolás Serván Eyzaguirre*
}

Resumen. - En el presente artículo, los autores preocupados por las inclusión de reglas propias del proceso civil en el arbitraje, comentan las mejores prácticas internacionales para la organización de un arbitraje, desde la presentación de memoriales escritos, pasando por la etapa probatoria y la exhibición de documentos, hasta la implementación de tecnología en los arbitrajes.

Abstract. - The authors, concerned about the inclusion of procedural rules of the civil process in the arbitration field, discuss the best international practices for the organization of an arbitration, ranging the presentation of written memorials, passing through the evidentiary stage and the disclosure of documents, and the implementation of technology in arbitration.

Palabras claves. - Arbitraje - Reglas procesales - Arbitrajes Ad Hoc - Buenas prácticas arbitrales - Organización del arbitraje.

Keywords. - Arbitration - Procedural rules - Ad Hoc Arbitration - Best practices in arbitration - Organization of the arbitration.

* Abogado por la Pontificia Universidad Católica del Perú y Master en Derecho (LL.M.) por la Universidad de Yale. Decano de la Facultad de Derecho de la Universidad del Pacífico. Profesor de Arbitraje Comercial y Arbitraje de las Inversiones. Árbitro nacional e internacional. Miembro del Board of Reporters del Institute of Transnacional Arbitration (ITA), del Grupo Latinoamericano de Arbitraje de la Cámara de Comercio Internacional (CCI), de la Asociación Americana de Derecho Internacional Privado (ASADIP), del Comité Editor de la Revista Peruana de Arbitraje, de la Association for International Arbitration (AIA), de la Asociación Latinoamericana de Arbitraje y del Comité Argentino de Arbitraje Nacional y Transnacional (CARAT). Miembro de la lista de árbitros de los principales Centros de Arbitraje del Perú y del Energy Arbitrators' list. Co.autor de la Ley de Arbitraje peruana de 2008. Moderador del ITA FORO DE ARBITRAJE LATINOAMERICANO (ITAFOR).

* Alumno de noveno ciclo de Facultad de Derecho la Universidad del Pacífico. Presidente del Consejo Directivo la Revista Forseti. 


\section{Introducción}

El proceso y el arbitraje son ambos mecanismos de resolución de controversias, pero ¿esto implica que el arbitraje debe desarrollarse como un proceso judicial? La respuesta es que no, porque precisamente una de las principales razones por las que las partes pactan un convenio arbitral para resolver sus controversias es la flexibilidad, frente al rito procesal derivado de un proceso. Gracias a esta flexibilidad, los aspectos procedimentales pueden adaptarse a las necesidades de las partes: "so long as the parties are treated fairly, an arbitration may be tailored to meet the specific requirements of the dispute, rather than conducted in accordance with fixed procedural rules"1. De ahí que, un arbitraje bien llevado es aquel en el que los árbitros conducen el arbitraje de acuerdo con las necesidades de las partes.

Un ejemplo nos permite resaltar la importancia de los aspectos procedimentales del arbitraje. A diferencia de lo que ocurre en el proceso judicial en el que el plazo para contestar la demanda viene establecido de manera previa por la ley de acuerdo con la vía procedimental predeterminada, en el arbitraje las partes y los árbitros cuentan con libertad para que, de acuerdo con las circunstancias del caso concreto, puedan establecer mayores o menores plazos para la presentación de una demanda o de una contestación de demanda. En el fondo, las partes someten sus controversias a arbitraje con el objetivo de obtener un trajo justo y procedimiento neutral, flexible, eficiente y susceptible de ser moldeado de acuerdo con las necesidades de las partes, sin referencia a las formalidades procedimentales de las Cortes nacionales ${ }^{2}$.

El principal problema que se presenta, sin embargo, es que los actores jurídicos y los árbitros no saben aprovechar esta y otras ventajas que ofrece el arbitraje para la resolución de conflictos. Como bien señala Kundmuller, "en vez de confirmarse en nuestro medio una paulatina y creciente especialización del arbitraje; lo que se observa es un síndrome del 'hijo pródigo'. El arbitraje se jurisdiccionaliza y procesaliza cada vez más, regresando al 'redil' de la jurisdiccionalidad estatal y dejando de ser una 'oveja descarriada' del Derecho"3.

En este contexto, este artículo tiene como principal objetivo identificar una serie de herramientas que le permitan a los actores jurídicos que participan del arbitraje combatir la "judicialización" del arbitraje.

La incorporación de estas "buenas prácticas" en el arbitraje permitirán aplicar de manera plena todas las ventajas que tiene nuestra Ley de Arbitraje. Para estos

1 BLACKABY, Niguel, PARTASIDES, Constantine, REDFERN, Alan y HUNTER, Martin. Redfern and Hunter on International Arbitration. Nueva York: Oxford University Press, 6ta edición, 2009, p. 30 .

2 BORN, Gary. International Commercial Arbitration. Austin: Aspen publishers, 2da edición, 2014, p. 1525.

3 KUNDMULLER, Franz. Es Urgente "Arbitrabilizar" al Arbitraje Peruano. Lima: Arbitraje PUCP, 2012, p. 62. Consultado el 30 de marzo en: http://revistas.pucp.edu.pe/index.php/arbitrajepucp/article/view/9358 
efectos, apelaremos a varias de estas "buenas prácticas" que ya se vienen utilizando en otras latitudes con éxito.

\section{Organización del Arbitraje}

No es un aspecto desconocido que al amparo de la Ley de Arbitraje, Decreto Legislativo $N^{\circ} 1071$ (en adelante, la "Ley de Arbitraje"), las partes pueden determinar libremente las reglas procedimentales aplicables al arbitraje. Ahora bien, por lo general si bien las partes tienen libertad para elegir las reglas que determinen más apropiadas para su caso concreto, es el tribunal arbitral el que se encarga de la dirección del procedimiento en el día a día, una vez que se encuentra constituido; sin embargo, esta dirección tiene que ser contrastada con las necesidades de las partes 4 .

Por tanto, lo primero que corresponde es que el tribunal arbitral emita una primera orden procesal que contemple los aspectos esenciales para desarrollar un arbitraje que se ajuste a la necesidad de las partes. A diferencia de lo que sucede en el proceso judicial, que es estándar para todos los casos que siguen una misma vía procedimental, el procedimiento arbitral puede y debe adaptarse a lo que requiere el caso concreto.

Y esta adaptación caso por caso, requiere, esencialmente, que el tribunal arbitral consulte siempre con las partes sobre aspectos específicos del procedimiento.

En efecto, las Notas de la CNUDMI sobre la organización del proceso arbitral (en adelante, "NOTAS ORGANIZACIÓN") destaca como una buena (e indispensable) práctica en el arbitraje las "consultas relativas a la organización del proceso arbitral" 5 , que se pueden llevar adelante tanto en forma presencial como remota (por ejemplo, intercambio de correos electrónicos o conferencias telefónicas) y cuyo resultado será la emisión de una orden procesal que contenga las reglas de juego del arbitraje en cuestión.

Estas reglas de juego que serán esenciales para el buen desempeño de un arbitraje, deberán considerar los principales eventos procedimentales en el curso de todo arbitraje ${ }^{6}$ :

4 BLACKABY, PARTASIDES, REDFERN y HUNTER. Op. cit.,, p. 364. Traducción libre de: “When the arbitral tribunal is established, day-to-day control of the proceeding begins to pass to the tribunal. However, the transfer of control is not total and is not immediate. The Tribunal usually engages in a dialogue with the parties on procedural matters, and often a 'Procedural Order No. 1' is issued to design the essential elements of the process and the time limits within which each stage is to take place".

5 NOTA ORGANIZACIÓN, Notas No 9 a la 13.

Se pueden consultar las Notas de la CNUDMI sobre la organización del proceso arbitral en: https://uncitral.un.org/sites/uncitral.un.org/files/media-documents/uncitral/es/arb-notes2016-ebook-s.pdf.

6 Ver: BORN, Gary. International Arbitration: Law and Practice. Países Bajos: Kluwer International Arbitration, 2012, p. 159. También, BLACKABY, PARTASIDES, REDFERN y HUNTER. Op. cit.,, p. 370 . 
Intercambio de memoriales
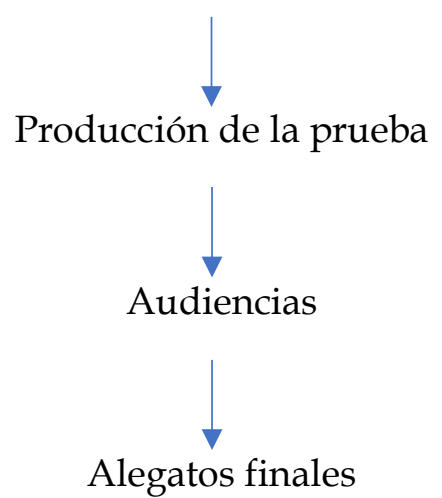

Tanto para las consultas a las partes, como para la adopción de la orden procesal que establezca las reglas que serán aplicables al arbitraje en cuestión, las NOTAS ORGANIZACIÓN resultan muy útiles porque en ellas se identifican los aspectos relevantes del procedimiento que deben ser considerados por el tribunal arbitral. Estos asuntos van desde los temas administrativos, como la notificación de documentos, hasta reglas sobre el material probatorio que se presentará.

Varias de estas reglas serán analizadas seguidamente como buenas prácticas que se deberán implementar en el arbitraje nacional para dotar de mayor eficiencia y eficacia los procedimientos arbitrales.

\section{i. La presentación de los memoriales o escritos}

La importancia de los memoriales o escritos de fondo radica en que permiten al tribunal arbitral identificar de manera clara los asuntos sobre los que tendrá que pronunciarse, al tiempo que las partes presentan sus argumentos de hecho y de derecho. Existen dos tipos de memoriales o escritos (que no incluyen la solicitud de arbitraje y su respuesta). Por un lado, están la demanda, la contestación y reconvención y su contestación (etapa postulatoria); y, por otro lado, los que sintetizan las posiciones de las partes, el material probatorio más relevante e incluyen una síntesis de lo sucedido en las audiencias ${ }^{7}$ (memoriales de cierre o, como se conoce en la jerga legal peruana, alegatos).

La determinación de la forma en la que se presentarán los escritos o memoriales sobre el fondo del caso es de trascendental importancia, pues marca el curso que seguirá el arbitraje. Esta debe adecuarse a lo requerido por las partes dependiendo de cada caso en concreto.

En los casos de menor envergadura es recomendable que el tribunal arbitral proponga que solamente se presente una ronda de escritos postulatorios. Mientras que, en los casos más grandes, debido al volumen de información que se suele

7 WAINCYMER, Jeffrey. Procedure and Evidence in International Arbitration. Países Bajos: Kluwer Law International, 2012, pp. 479-480. 
presentar y los extensos argumentos, se podrían acordar dos rondas de escritos cruzados. Una decisión adicional que se debe adoptar luego de escuchadas las partes, es si la presentación de escritos será consecutiva y alternada entre las partes, como sucede en la mayoría de los casos de tal forma que se puedan responder todos los puntos o, por el contrario, si los memoriales serán presentados de manera simultánea ${ }^{8}$. En caso se elija la segunda opción, se recomienda optar por un intercambio de escritos, a fin de que se puedan contestar todos los argumentos planteados por la contraparte 9 .

Una vez que el tribunal arbitral ha determinado, luego de consultar con las partes, la forma en la que se presentarán los escritos de fondo, lo siguiente es determinar el cronograma de esa presentación, a fin de mantener el control del curso del arbitraje y evitar prolongaciones innecesarias.

Como bien señala Born:

"As a practical matter, the content, form and timing of written submission vary. In some arbitration (particularly smaller ones), written submissions are brief, informal documents submitted shortly before the evidentiary hearing; most of the parties' submissions will be oral, made at hearing itself. In other arbitration (typically larger disputes), written submissions will require several months to prepare and will be hundreds of pages long (not including exhibits, which will entail thousands of additional pages). The timetables adopted for arbitration will obviously vary substantially; depending on whether the written submissions fall closer to one end of this spectrum or the other" 10 .

Además, deberá considerarse si será de aplicación plena lo dispuesto en el artículo 39 de la Ley de Arbitraje que permite la ampliación o modificación de la demanda11, en cuyo caso será importante prever cómo será la nueva ronda de escritos y sus tiempos. En todo caso, resulta apropiado consultar con las partes si resulta pertinente establecer una fecha máxima en la que se podrá modificar o ampliar la demanda.

También debe consultarse (y luego establecer en la orden procesal respectiva) lo relativo a una eventual ampliación de plazo para la presentación de los memoriales sobre el fondo de la controversia ${ }^{12}$. Si no existe pacto al respecto, el tribunal arbitral deberá aplicar lo establecido en el inciso 4 del artículo 34 de la Ley

8 NOTA ORGANIZACIÓN, Nota No 66

9 Nota $\mathrm{N}^{\mathrm{o}} 41$.

10 BORN, Gary. International Arbitration: Law and Practice. Países Bajos: Kluwer International Arbitration, 2012, p.165.

11 Artículo 39(3) de la Ley de Arbitraje: “3. Salvo acuerdo en contrario, en el curso de las actuaciones, cualquiera de las partes podrá modificar o ampliar su demanda o contestación [...]".

12 LEW, Julian; MISTELIS, Louka. y KRÖLL, Stefan. Comparative International Commercial Arbitration. Países Bajos: Wolters Kluwer Law \& Business, 2003, p. 541. “Tribunals will invariably fix time limits for the submission of written pleadings. Time limits set be the tribunal should be seen as "target time limits" (77) rather than deadlines; they should be extended when it is reasonable and fair to do so. When deciding whether to admit late submissions a tribunal should consider the circumstances of the cases, the need for equality and fairness, the possibility of prejudice to the other party and the requirements for orderly conduct of proceedings". 
de Arbitraje, así "El tribunal arbitral podrá, a su criterio, ampliar los plazos que haya establecido para las actuaciones arbitrales, incluso si estos plazos estuvieran vencidos".

Por último, terminada la audiencia de pruebas es común la presentación de memoriales de cierre (alegatos escritos) que resumen los argumentos de las partes. Estos pueden presentarse de manera previa a una audiencia final (que en el argot peruano llamamos audiencia de informes orales) o, si existe solamente una audiencia de pruebas concentrada (que suele culminar con alegaciones orales finales de las partes), los alegatos escritos pueden presentarse después de esta. También debe considerarse si la presentación de los alegatos escritos será simultánea o consecutiva y si habrá una o dos rondas de escritos.

En cualquier caso, los árbitros deben tener en consideración las circunstancias específicas de la disputa para dotar de la mayor eficiencia posible la presentación de escritos por las partes; así como transmitir a las partes su intención sobre la forma en la que se debe desarrollar el proceso.

\section{ii. Presentación de documentos en poder de cada una de las partes}

Un aspecto esencial de cualquier arbitraje es la parte probatoria. De ahí que, debe preverse en la primera orden procesal (luego de consultar con las partes) si el material probatorio de carácter documentario en poder de cada una de las partes deberá ser presentado junto con los memoriales postulatorios (que es lo óptimo) o si se podrán presentar en un momento posterior, en cuyo caso será conveniente establecer plazos máximos o, en todo caso, condiciones para dicho ofrecimiento, que de no observarse conllevará a la no admisión de la prueba por tardía o extemporánea.

También debe consultarse y luego regularse, la forma en que se presentará la documentación (en físico o en base digital o ambos) ${ }^{13}$.

A fin de determinar todo lo referente a la admisión de la prueba, resulta más que pertinente acordar con las partes la adopción de las Reglas de la IBA (International Bar Association) sobre práctica de la prueba en el arbitraje internacional, que constituye un instrumento de soft law que puede resultar de gran ayuda. Así, el tribunal arbitral puede establecer desde la Orden Procesal $N^{\circ} 1$, que las únicas objeciones válidas para oponerse a un medio probatorio serán las establecidas en el artículo 9 de las Reglas de la IBA:

“El Tribunal Arbitral podrá excluir, a instancia de parte o de oficio, la prueba o la exhibición de cualquier Documento, declaración, testimonio oral o inspección por cualquiera de las siguientes razones:

13 WAINCYMER. Op. cit., p. 454. 
(a) Falta de relevancia suficiente o utilidad para la resolución del caso;

(b) existencia de impedimento legal o privilegio bajo las normas jurídicas o éticas determinadas como aplicables por el Tribunal Arbitral;

(c) onerosidad o carga excesiva para la práctica de las pruebas solicitadas;

(d) pérdida o destrucción del Documento, siempre que se demuestre una razonable probabilidad de que ello haya ocurrido;

(e) confidencialidad por razones comerciales o técnicas que el Tribunal Arbitral estime suficientemente relevantes;

(f) razones de especial sensibilidad política o institucional que el Tribunal Arbitral estime suficientemente relevantes (incluyendo pruebas que hayan sido clasificadas como secretas por parte de un gobierno o de una institución pública internacional); o

(g) consideraciones de economía procesal, proporcionalidad, justicia o igualdad entre las Partes que el Tribunal Arbitral estime suficientemente relevantes".

La adopción de estas reglas debe ir acompañada de plazos claros y precisos para oponerse a tal o cual prueba. No debemos olvidar que en caso el plazo se exceda, nos encontramos frente a una renuncia del derecho a objetar ${ }^{14}$.

Además, como bien se señala en las NOTAS ORGANIZACIÓN, es conveniente que se establezcan asunciones como las siguientes en caso no se presente objeción respecto a algún documento: “a) todo documento procede de la fuente en él indicada, b) todo ejemplar de una comunicación expedida (por ejemplo, por carta, télex, telefax o algún otro medio electrónico) ha sido recibido por su destinatario, sin necesidad de otra prueba, y c) toda copia es conforme"15. Esto puede darle mayor celeridad al arbitraje y evitar dilaciones innecesarias.

\section{iii. Solicitud de exhibición de documentos}

La revelación de documentos, conocida también como disclosure of documents, es una técnica muy desarrollada en el common law que consiste en la solicitud de determinados tipos de documentos en poder de la contra parte ${ }^{16}$. Si bien es cierto en el derecho anglosajón las partes esencialmente construyen sus casos a partir de esta revelación de documentos, nada impide aplicar esta técnica en sistema del civil law, basados en la buena fe que debe regir la etapa probatoria.

En efecto, especialmente en el arbitraje internacional su aplicación ha ido en aumento ${ }^{17}$.

4 Artículo 11 de la Ley de Arbitraje del Perú.

15 NOTA ORGANIZACIÓN, Nota No 52.

16 FERNÁNDEZ ROZAS, José Carlos. Tratado del arbitraje comercial en América Latina. Madrid: Editorial Iustel, 2008, p. 761.

17 EMANUELE, Ferdinando; MOLFA, Milo y JEDREY, Nathaniel. Evidence in International Arbitration: The Italian Perspective and Beyond. Nueva York: Thomson Reuters, 2016, p. 6. 
A diferencia de lo que suele ser la práctica arbitral en el Perú18, en el que contagiados por la práctica judicial, la exhibición de documentos es muy limitada, cada vez más se utiliza el artículo 3 de las Reglas de la IBA sobre práctica de pruebas, que señala cuales son los requisitos indispensables que debe contener toda solicitud de revelación de documentos:

“Una Solicitud de Exhibición de Documentos deberá contener:

(a) (i) una descripción de cada Documento cuya exhibición se solicite que sea su cliente para identificarlo, $\mathrm{o}$

(ii) una descripción suficientemente detallada (incluyendo el asunto de que se trate) de la concreta y específica categoría de Documentos requeridos que razonablemente se crea que existen; en el caso de Documentos conservados en formato electrónico, la Parte solicitante puede o el Tribunal Arbitral puede requerirle que proceda a, identificar archivos específicos, términos de búsqueda, individuos o cualquier otro medio de búsqueda para esos Documentos en una forma eficiente y económica.

(b) una declaración de por qué los Documentos requeridos son relevantes para el caso y sustanciales para su resolución; y

(c) una declaración de que los Documentos requeridos no se encuentran en poder, custodia o control de la Parte que los solicita o una declaración de las razones por las cuales sería irrazonablemente gravoso para la Parte solicitante exhibir tales Documentos, y

(d) una declaración sobre las razones por las cuales la Parte solicitante supone que los Documentos requeridos están en poder, custodia o control de otra Parte".

A efectos de implementar con éxito esta técnica, resulta esencial que el tribunal arbitral acuerde con las partes la oportunidad en la que éstas (de manera sucesiva o simultánea) solicitarán la exhibición de documentos.

Seguidamente, a efectos de garantizar eficiencia y orden, debe acordarse utilizar el "Redfern Schedule"19. El beneficio tangible de la utilización del Redfern Schedule es que permite al tribunal arbitral analizar los argumentos de las partes sobre por qué debe o no revelarse tal o cual documento y adoptar la respectiva decisión en ese mismo formato (ver un ejemplo en el Anexo 1).

18 Que felizmente poco a poco va cambiando gracias a la utilización de buenas prácticas como las que describiremos seguidamente y que, por ejemplo, la Cámara de Comercio de Lima las promueva.

19 El Centro Internacional de Arreglo de Diferencias Relativas a Inversiones (CIADI - ICSID) publica en su web el siguiente ejemplo del Redfern Schedule: https://icsid.worldbank.org/en/Documents/process/Redfern.doc

Adicionalmente, ver: GRIERSON, Jacob \& VAN HOOFT, Annet. Arbitrating under the 2012 ICC Rules. Países Bajos: Kluwer Law International, 2012, p. 176. 
También el tribunal arbitral deberá establecer en su orden procesal cuáles serán los motivos que adoptará como razonables al momento de resolver respecto a alguna objeción a exhibir. Como bien señala la doctrina, "las objeciones de parte pueden ser muy variadas, pero pueden dividirse en dos categorías: a) objeciones que justifican la denegatoria total; $y$ b) objeciones que pueden abordarse mediante la reducción de la solicitud o la adopción de otras medidas" 20.

Generalmente, se suelen aplicar las propias Reglas de la IBA sobre práctica de pruebas, en específico su artículo 9.2 que ha sido transcrito párrafos arriba ${ }^{21}$.

Por último, en caso no se produzca la revelación de documentos solicitada y no se expresen objeciones que resulten razonables para el tribunal arbitral, las reglas de la IBA permiten aplicar inferencias adversas o negativas. Esto quiere decir que el tribunal arbitral puede considerar que los documentos no producidos son contrarios a los intereses de la parte que se negó injustificadamente a presentarlos 22 .

\section{iv. Los testigos de hechos}

Es practica arraigada en los arbitrajes peruanos que, al igual de lo que sucede en el campo judicial (nuevamente contaminados), los testigos de hechos sean interrogados directamente en audiencia.

En cambio, es una buena práctica en el arbitraje que los testigos ofrecidos por cada parte y que, además, sean controlados por ellas, presenten sus testimonios por escrito acerca de los hechos que declaran ${ }^{23}$. Esto resulta de mucha utilidad, ya que, por ejemplo, le permite al abogado de la contraparte analizar si es que conviene o no citar a interrogatorio en audiencia a tal o cual testigo y, de resultar afirmativa la respuesta, tener tiempo suficiente para preparar el interrogatorio.

Corresponde pues que las partes y el tribunal arbitral coordinen temas como: (i) oportunidad en la que las partes ofrecerán a sus testigos, así como la oportunidad en la que se presentarán sus declaraciones escritas; (ii) oportunidad en la que se podrá plantear alguna objeción; (iii) oportunidad en la que las partes harán saber a cuál testigo ofrecido por su contraparte desean interrogar en audiencia.

También deberá decidirse acerca de la manera como se llevarán adelante los interrogatorios. De acuerdo con Lew, Mistelis y Kröll, "generalmente, una combinación de procedimientos inquisitoriales y contradictorios es la mejor solución. Incluso cuando se sigue un procedimiento de inquisitorio, es importante que los asuntos que deben formar la base de la decisión del tribunal arbitral estén disponibles para el examen y análisis crítico por las partes" 24 .

\footnotetext{
EMANUELE, MOLFA y JEDREY. Op. cit., p. 75.

Artículo 3.5 de las Reglas de la IBA sobre práctica de la prueba en el arbitraje internacional.

Artículo 9.2 de las Reglas de la IBA.

BLACKABY, PARTASIDES, REDFERN y HUNTER. Op. cit., p. 402.

LEW, MISTELIS y KRÖLL. Op. cit., p. 533.
} 
Un tema adicional que conviene precisar en la orden procesal, es si las partes (más precisamente sus asesores legales) puedan preparar a los testigos antes del interrogatorio. Si bien se trata de una actividad arraigada y hoy plenamente aceptada, es importante destacar que existen ciertos límites como, por ejemplo, que los abogados no deben escribir las declaraciones de los testigos o que los instruyan para que presenten ante el tribunal arbitral declaraciones abiertamente falsas ${ }^{25}$. Sin perjuicio de que comúnmente se resalta que la evidencia documental es preferida o tiene un peso superior a la evidencia de testigos ${ }^{26}$, el tribunal arbitral tiene libertad en la valoración de las pruebas.

\section{v. La Audiencia}

La judicialización del arbitraje en el Perú y la falta de expertos en esta materia, ha tenido como consecuencia de que se vuelva costumbre la realización de muchas audiencias durante plazos muy largos.

Es cierto que la Ley de Arbitraje regula el principio de libertad de audiencias bajo la premisa de que "el tribunal arbitral decidirá si han de celebrarse audiencias para la presentación de alegaciones, la actuación de pruebas y la emisión de conclusiones, o si las actuaciones serán solamente por escrito" 27; sin embargo, ello no significa que realizar muchas audiencias probatorias sea lo óptimo.

Las NOTAS ORGANIZACIÓN justamente se plantean la interrogante sobre si conviene realizar una sola audiencia (conocida como audiencia concentrada) o, si por el contrario, es más adecuado realizar varias audiencias separadas ${ }^{28}$.

Una sola audiencia concentrada destaca claramente. No solo reducirá significativamente los tiempos y costos del arbitraje, sino que además, y principalmente, permitirá a las partes presentar de manera completa y comprensiva su caso frente al tribunal arbitral. Es más, generalmente esta audiencia suele empezar con unos alegatos de apertura de las partes $\mathrm{y}$, luego de actuada toda la prueba, se concluye la audiencia con un alegato oral de cierre de las partes.

Una vez adoptada la decisión acerca de la audiencia, es conveniente programar preliminarmente un espacio de tiempo en el que se podrá llevar adelante la misma, con la finalidad de evitar problemas de agenda tanto del tribunal arbitral, como de las partes. Además, el conocimiento previo de esta fecha permitirá a las partes coordinar disponibilidad de los peritos y testigos.

Una vez que se culmina la parte postulatoria del arbitraje y se conoce la complejidad del caso, el número de testigos y peritos a interrogar, entre otros,

25 BLACKABY, PARTASIDES, REDFERN y HUNTER. Op. cit., p. 403.

26 BORN. Op. cit., p. 2256.

27 Artículo 42.1 de la Ley de Arbitraje

28 NOTA ORGANIZACIÓN, Nota N 76. 
como bien señala Born, lo que corresponde como buena práctica es que el tribunal arbitral de directrices sobre el desarrollo de esta audiencia:

"Prior to hearing, and after consultation with the parties, the tribunal will typically issue procedural directions for organization of the hearing. The directions will fix the length of the hearing (...), the order of oral submissions, the order of witnesses and (ordinarily) the estimated time for counsels' oral statements and witness examination" 29 .

Una buena práctica sobre la base del principio de equidad entre las partes, es que el tribunal arbitral determine (previa consulta con las partes) la misma cantidad de tiempo para las partes. Las NOTAS ORGANIZACIÓN señalan que la determinación del tiempo "facilitará la planificación por las partes de la presentación de sus diversas pruebas y alegaciones, reducirá la posibilidad de que al final de las vistas falte tiempo, y evitará que algunas de las partes haga un empleo desmesurado de éste" 30 . Esto también va de la mano con el orden en el que los testigos y peritos serán interrogados.

Finalmente, es recomendable que al momento de adoptar cualquier decisión procedimental sobre las audiencias, el tribunal arbitral consulte a las partes vía telefónica o por correo electrónico a fin de adaptar cualquier decisión a sus requerimientos y las necesidades del caso en concreto. Por ejemplo, consultar si el tribunal arbitral va a aceptar notas o síntesis de las posiciones de las partes en la audiencia, que permitan identificar las principales discusiones tanto de hecho como de derecho planteadas.

\section{vi. Apelar a la tecnología y alejarse lo más posible de las prácticas judiciales}

El rito procesal de que la comunicación entre el juez y las partes se realiza mediante escritos y resoluciones, puede ser superada por medio de la flexibilidad del arbitraje. Tal como señalan las NOTAS ORGANIZACIÓN: "podrá convenirse en intercambiar documentos, o algunos de ellos, no sólo sobre soporte de papel sino también en alguna forma electrónica distinta del telefax (por ejemplo, por correo electrónico, o en discos magnéticos u ópticos), o bien únicamente en forma electrónica" ${ }^{\prime 3}$.

En ese contexto, si los reglamentos arbitrales aplicables lo permiten ${ }^{32} \mathrm{o}$ los árbitros lo establecen con la venia de las partes, las comunicaciones podrán producirse de manera electrónica. Esto significa que las partes remitirán sus memoriales por correo, al tiempo que el tribunal arbitral podrá notificar sus decisiones también por esa misma vía. Así la cercanía de los árbitros con el curso del procedimiento arbitral será mucho mayor.

29 BORN, Gary. International arbitration: Law and practice. Alphen Van den Rijn: Kluwer Law International, 2012, p. 167.

30 NOTA ORGANIZACIÓN, Nota N ${ }^{\circ} 79$.

31 Nota No 36.

32 Por ejemplo, el reglamento del Centro de Arbitraje de la Cámara de Comercio de Lima de 2017. 
Finalmente, es importante señalar que a diferencia de lo que sucede en el proceso civil donde el juez suele pronunciarse sobre cada uno de los escritos de las partes, esto no es necesario en el arbitraje. Algunas partes y sus abogados esperan, innecesariamente, por ejemplo, que el tribunal arbitral declare expresamente que admite cada uno de los medios probatorios, a pesar que no se han emitido objeciones sobre ellos. Tampoco es necesario que el tribunal arbitral se pronuncie para correr traslado de los escritos presentados por las partes, ya que simplemente una vez notificado el escrito empieza a correr el plazo establecido en la Orden Procesal $N^{o} 1$ para contestar, de ser necesario o ser de interés de la parte ${ }^{33}$.

\section{Conclusiones}

La única forma de que el Perú se convierta en una plaza atractiva para la práctica del arbitraje en la región, es dotar al procedimiento arbitral de verdadera eficiencia y eficacia, tomando en cuenta las buenas prácticas arbitrales. Por el contrario, si persistimos en incorporar figuras propias del rito procesal judicial al arbitraje, terminaremos por destruir una institución que ostenta como una de sus principales banderas la flexibilidad procesal.

Por ello, cualquier tribunal arbitral que pretenda organizar de manera seria y profesional un arbitraje, que se ajuste a los requerimientos específicos de las partes y a la complejidad de la materia en controversia, debe guiarse de las NOTAS ORGANIZACIÓN. Claro está, que la decisión final sobre los aspectos procedimentales siempre deberá considerar los requerimientos de las partes. De ahí que, resulta más que necesaria una conferencia telefónica o hasta incluso una audiencia para la determinación de las reglas (en casos complejos).

A veces se piensa que la única labor del árbitro consiste en la emisión de un laudo ejecutable. Sin duda, esa es una de las tareas más importantes. Sin embargo, otra por demás relevante consiste en que el tribunal arbitral tenga la capacidad de manejar el procedimiento de manera eficiente en términos de garantizar el derecho de defensa de las partes, así como en términos económicos. Todo buen arbitraje, por lo tanto, comienza con la emisión de una Orden Procesal $N^{\circ} 1$ que recoja todos los aspectos procedimentales esenciales.

33 Es más, si el tribunal arbitral tiene interés en una respuesta, lo hará saber a las partes dando un plazo para ello. 


\section{Anexo $\mathrm{N}^{\circ} 1$ Redfern Schedule}

\begin{tabular}{|c|c|c|c|c|c|}
\hline $\begin{array}{c}\text { Solicitud } \\
\mathrm{N}^{\mathrm{o}}\end{array}$ & $\begin{array}{l}\text { Documentos } \\
\text { o categoría } \\
\text { de } \\
\text { documentos } \\
\text { solicitados }\end{array}$ & $\begin{array}{c}\text { Relevancia y } \\
\text { materialidad } \\
\text { de los } \\
\text { documentos } \\
\text { solicitados }\end{array}$ & $\begin{array}{c}\text { Objeciones a } \\
\text { la solicitud } \\
\text { de } \\
\text { documentos } \\
\text { presentada }\end{array}$ & $\begin{array}{l}\text { Respuesta a } \\
\text { la objeción }\end{array}$ & $\begin{array}{c}\text { Decisión del } \\
\text { Tribunal }\end{array}$ \\
\hline 1. & $\begin{array}{c}\text { Todas las } \\
\text { actas de la } \\
\text { junta } \\
\text { general de } \\
\text { accionista de } \\
\text { la empresa X } \\
\text { desde el } \\
2015 \text { a la } \\
\text { fecha. }\end{array}$ & $\begin{array}{c}\text { La } \\
\text { solicitudes } \\
\text { relevante } \\
\text { debido a } \\
\text { que en esas } \\
\text { sesiones se } \\
\text { ha discutido } \\
\text { la relación } \\
\text { comercial } \\
\text { con la } \\
\text { empresa Y. }\end{array}$ & $\begin{array}{c}\text { La relevación } \\
\text { de } \\
\text { documentos } \\
\text { solicitada no } \\
\text { es relevante } \\
\text { puesto que } \\
\text { escapa de la } \\
\text { materia en } \\
\text { controversia, } \\
\text { al mismo } \\
\text { tiempo que } \\
\text { afecta el } \\
\text { secreto } \\
\text { comercial de } \\
\text { la empresa X } \\
\text { y es } \\
\text { altamente } \\
\text { costosa. }\end{array}$ & $\begin{array}{c}\text { La objeción } \\
\text { planteada } \\
\text { carece de } \\
\text { asidero legal. } \\
\text { Primero, } \\
\text { porque los } \\
\text { documentos } \\
\text { solicitados } \\
\text { servirán para } \\
\text { probar los } \\
\text { hechos de las } \\
\text { pretensiones } \\
\text { que } \\
\text { planteamos } \\
\text { en la medida } \\
\text { que acredita } \\
\text { la intención } \\
\text { de la } \\
\text { compañía X } \\
\text { de adquirir } \\
\text { acciones de } \\
\text { la compañía } \\
\text { Y. }\end{array}$ & $\begin{array}{c}\text { El tribunal } \\
\text { ordena la } \\
\text { producción } \\
\text { de } \\
\text { documentos } \\
\text { de } \\
\text { solamente } \\
\text { aquellas } \\
\text { actas que } \\
\text { abordan } \\
\text { asuntos } \\
\text { relacionados } \\
\text { con la } \\
\text { empresa Y. }\end{array}$ \\
\hline
\end{tabular}

